\title{
Controlled Synthesis of Peptide-Based Amphiphilic Copolymers
}

\author{
Ivaylo V. Dimitrov ${ }^{*}{ }^{\dagger}$, Iliyana V. Berlinova ${ }^{\dagger}$, Plamen V. Iliev ${ }^{\dagger}$, Nikolay G. Vladimirov ${ }^{\ddagger}$ \\ ${ }^{\dagger}$ Institute of Polymers, Bulgarian Academy of Sciences, Sofia 1113, Bulgaria, \\ ${ }^{\sharp}$ Hercules Incorporated, Research Center, Wilmington, Delaware, 19808-1599, USA
}

\section{Supporting Information}

1. Characterization of methoxypoly(oxyethylene) $\alpha$-naphthylmaleamate (IIa-2):

Yield: $86 \% .{ }^{1} \mathrm{H}$ NMR $\left(\mathrm{CDCl}_{3}\right): \delta 3.37\left(\mathrm{CH}_{3}-\mathrm{O}\right) ; \delta 3.64\left(\mathrm{O}-\mathrm{CH}_{2}-\mathrm{CH}_{2}-\mathrm{O}\right) ; \delta 4.33\left(\mathrm{CH}_{2}-\mathrm{O}-\mathrm{C}=\mathrm{O}\right) ; \delta$ 6.64-6.85 (CH=CH+ $\mathrm{CH}^{1}$-Naphthyl); $\delta$ 7.27-8.15 ( $\mathrm{CH}^{2-7}$-Naphthyl); $\delta 8.95(H \mathrm{~N}-\mathrm{C}=\mathrm{O})$.

2. Characterization of PEO-based macroinitiators.

2.1. Macroinitiator IIIa-2 (Michael addition of AET.HCl to methoxypoly(oxyethylene) $\alpha$ naphthylmaleamate):

Yield: $75 \%$. GPC in $N, N$-dimethylacetamide (vs. PEO standards): $M_{\mathrm{w}} / M_{\mathrm{n}}=1.26 .{ }^{1} \mathrm{H} \mathrm{NMR}\left(\mathrm{CDCl}_{3}\right)$ : $\delta 2.85\left(\mathrm{~S}-\mathrm{CH}_{2}\right) ; \delta 3.00-3.15\left(\mathrm{CH}_{2}-\mathrm{N}^{+}+\mathrm{O}=\mathrm{C}-\mathrm{CH}_{2}-\mathrm{CH}\right) ; \delta 3.37\left(\mathrm{CH}_{3}-\mathrm{O}\right) ; \delta 3.64\left(\mathrm{O}-\mathrm{CH}_{2}-\mathrm{CH}_{2}-\mathrm{O}\right) ; \delta 4.33$ $\left(\mathrm{CH}_{2}-\mathrm{O}-\mathrm{C}=\mathrm{O}\right) ; \delta 4.45(\mathrm{O}=\mathrm{C}-\mathrm{CH}-\mathrm{S}) ; \delta 4.45(\mathrm{O}=\mathrm{C}-\mathrm{CH}-\mathrm{S}) ; \delta 6.65\left(\mathrm{CH}^{1}\right.$-Naphthyl $) ; \delta 7.27-8.15\left(\mathrm{CH}^{2-7}-\right.$ Naphthyl); $\delta 8.95(H \mathrm{~N}-\mathrm{C}=\mathrm{O})$. 
2.2. Macroinitiator IIIa-3 (Michael addition of Cys-OEt.HCl to methoxypoly(oxyethylene) dodecylmaleamate):

Yield: $72 \%$. GPC in $N, N$-dimethylacetamide (vs. PEO standards): $M_{\mathrm{w}} / M_{\mathrm{n}}=1.34 .{ }^{1} \mathrm{H} \mathrm{NMR}\left(\mathrm{CDCl}_{3}\right)$ : $\left.\delta 0.87\left(\mathrm{CH}_{3}\right) ; \delta 1.15-1.40\left(\mathrm{CH}_{3}-\left(\mathrm{CH}_{2}\right)_{9}\right)+\mathrm{CH}_{3}-\mathrm{CH}_{2}-\mathrm{O}\right) ; \delta 1.50\left(\left(\mathrm{CH}_{2}\right)_{9}-\mathrm{CH}_{2}\right) ; \delta 2.90-3.25\left(\mathrm{O}=\mathrm{C}-\mathrm{CH}_{2}-\right.$ $\left.\mathrm{CH}+\mathrm{CH}_{2}-\mathrm{NH}+\mathrm{S}-\mathrm{CH}_{2}-\mathrm{CH}\right) ; \delta 3.37\left(\mathrm{CH}_{3}-\mathrm{O}\right) ; \delta 3.65\left(\mathrm{O}-\mathrm{CH}_{2}-\mathrm{CH}_{2}-\mathrm{O}\right) ; \delta 3.95(\mathrm{O}=\mathrm{C}-\mathrm{CH}-\mathrm{S}) ; \delta 4.16-4.35$ $\left(\mathrm{CH}_{2}-\mathrm{O}-\mathrm{C}=\mathrm{O}+\mathrm{O}-\mathrm{CH}_{2}-\mathrm{CH}_{3}\right) ; \delta 4.45\left(\mathrm{CH}-\mathrm{N}^{+}\right) ; \delta 8.25(\mathrm{HN}-\mathrm{C}=\mathrm{O})$.

3. Amphiphilic PEO-peptide block copolymers.

In a typical polymerization procedure, $0.4081 \mathrm{~g}(0.17 \mathrm{mmol})$ of the macroinitiator (IIIa-1) were dissolved in $10 \mathrm{~mL}$ of DMF and degassed. Separately, a solution of ZLLys-NCA (1.561 g, $5.1 \mathrm{mmol})$ in $10 \mathrm{~mL}$ of DMF was prepared and degassed. The two solutions were combined via transfer needle under dry argon and the polymerization was performed at $70{ }^{\circ} \mathrm{C}$ for 5 days. The polymer solution was concentrated and the product was precipitated in diethyl ether. Then it was extracted with methanol.

3.1. Block copolymer IVa-1.1 (obtained from macroinitiator IIIa-1):

Yield: $68 \%$. GPC in $N, N$-dimethylacetamide (vs. PEO standards): $M_{\mathrm{w}} / M_{\mathrm{n}}=1.38 .{ }^{1} \mathrm{H}$ NMR (DMSO$\left.d_{6}\right): \delta 0.81\left(\mathrm{CH}_{3}-\left(\mathrm{CH}_{2}\right)_{10}\right) ; \delta$ 1.10-2.00 $\left(\mathrm{CH}_{3}-\left(\mathrm{CH}_{2}\right)_{10}+\alpha \mathrm{CH}-\left(\mathrm{CH}_{2}\right)_{3}\right) ; \delta$ 2.80-3.10 $\left(\mathrm{S}-\mathrm{CH} H_{2}+\alpha \mathrm{CH}-\right.$ $\left.\left(\mathrm{CH}_{2}\right)_{3} \mathrm{CH}_{2}+\mathrm{O}=\mathrm{C}-\mathrm{CH}_{2}-\mathrm{CH}+\left(\mathrm{CH}_{2}\right)_{10}-\mathrm{CH}_{2}-\mathrm{NH}+\mathrm{S}-\mathrm{CH}_{2}-\mathrm{CH}_{2}-\mathrm{NH}\right) ; \delta 3.22\left(\mathrm{CH}_{3}-\mathrm{O}\right) ; \delta 3.50\left(\mathrm{O}-\mathrm{CH}_{2}-\right.$ $\left.\mathrm{CH}_{2}-\mathrm{O}+\mathrm{O}=\mathrm{C}-\mathrm{CH}-\mathrm{S}\right) ; \delta 3.75-4.36\left(\alpha \mathrm{CH}-\mathrm{NH}+\mathrm{CH}_{2}-\mathrm{O}-\mathrm{C}=\mathrm{O}\right) ; \delta 4.97\left(\mathrm{Z}-\mathrm{CH}_{2}\right) ; \delta 7.05-7.35\left(\mathrm{C}_{6} H_{5}+\right.$ $\left.\alpha \mathrm{CH}-\left(\mathrm{CH}_{2}\right)_{4}-\mathrm{N} H+\mathrm{S}-\left(\mathrm{CH}_{2}\right)_{2}-\mathrm{N} H\right) ; \delta$ 7.69-8.27 $\left(\alpha \mathrm{CH}-\mathrm{NH}+\mathrm{CH}_{2}-\mathrm{N} H-(\mathrm{C}=\mathrm{O})-\mathrm{CH}-\mathrm{S}\right)$.

3.2. Block copolymer IVa-2.1 (obtained from macroinitiator IIIa-2):

Yield: $73 \%$. GPC in $N, N$-dimethylacetamide (vs. PEO standards): $M_{\mathrm{w}} / M_{\mathrm{n}}=1.33 .{ }^{1} \mathrm{H}$ NMR (DMSO$\left.d_{6}\right): \delta 1.00-2.15\left(\alpha \mathrm{CH}-\left(\mathrm{CH}_{2}\right)_{3}\right) ; \delta 2.74-3.11\left(\mathrm{~S}-\mathrm{CH}_{2}+\alpha \mathrm{CH}-\left(\mathrm{CH}_{2}\right)_{3} \mathrm{CH}_{2}+\mathrm{O}=\mathrm{C}-\mathrm{CH}_{2}-\mathrm{CH}+\mathrm{S}-\mathrm{CH}_{2}-\mathrm{CH}_{2}-\right.$ $\mathrm{NH}) ; \delta 3.23\left(\mathrm{CH}_{3}-\mathrm{O}\right) ; \delta 3.50\left(\mathrm{O}-\mathrm{CH}_{2}-\mathrm{CH}_{2}-\mathrm{O}\right) ; \delta 3.64-4.43\left(\alpha \mathrm{CH}-\mathrm{NH}+\mathrm{CH}_{2}-\mathrm{O}-\mathrm{C}=\mathrm{O}+\mathrm{O}=\mathrm{C}-\mathrm{CH}-\mathrm{S}\right) ; \delta$ $4.94\left(\mathrm{Z}-\mathrm{CH}_{2}\right) ; \delta 6.65-8.93\left(\mathrm{C}_{6} \mathrm{H}_{5}+\alpha \mathrm{CH}-\left(\mathrm{CH}_{2}\right)_{4}-\mathrm{NH}+\mathrm{S}-\left(\mathrm{CH}_{2}-\mathrm{CH}-\mathrm{NH}+\mathrm{C}_{10} \mathrm{H}_{7}+\alpha \mathrm{CH}-\mathrm{NH}+\mathrm{C}_{10} \mathrm{H}_{7}-\right.\right.$ $\mathrm{N} H-\mathrm{C}=\mathrm{O})$. 
3.3. Block copolymer IVa-3.1 (obtained from macroinitiator IIIa-3):

Yield: $67 \%$. GPC in $N, N$-dimethylacetamide (vs. PEO standards): $M_{\mathrm{w}} / M_{\mathrm{n}}=1.36 .{ }^{1} \mathrm{H}$ NMR (DMSO$\left.d_{6}\right): \delta 0.81\left(\mathrm{CH}_{3}-\left(\mathrm{CH}_{2}\right)_{10}\right) ; \delta 1.00-2.10\left(\mathrm{CH}_{3}-\left(\mathrm{CH}_{2}\right)_{10}+\mathrm{CH}_{3}-\mathrm{CH}_{2}-\mathrm{O}+\alpha \mathrm{CH}-\left(\mathrm{CH}_{2}\right)_{3}\right) ; \delta 2.75-3.12\left(\mathrm{~S}-\mathrm{CH}_{2}\right.$ $\left.+\alpha \mathrm{CH}-\left(\mathrm{CH}_{2}\right)_{3} \mathrm{CH}_{2}+\mathrm{O}=\mathrm{C}-\mathrm{CH}_{2}-\mathrm{CH}+\left(\mathrm{CH}_{2}\right)_{10}-\mathrm{CH}_{2}-\mathrm{NH}\right) ; \delta 3.22\left(\mathrm{CH}_{3}-\mathrm{O}\right) ; \delta 3.50\left(\mathrm{O}-\mathrm{CH}_{2}-\mathrm{CH}_{2}-\mathrm{O}\right) ; \delta$ 3.62-4.33 $\left(\alpha \mathrm{CH}-\mathrm{NH}+\mathrm{CH}_{2}-\mathrm{O}-\mathrm{C}=\mathrm{O}+\mathrm{S}-\mathrm{CH}_{2}-\mathrm{CH}-\mathrm{NH}+\mathrm{O}=\mathrm{C}-\mathrm{CH}-\mathrm{S}+\mathrm{CH}_{3}-\mathrm{CH}_{2}-\mathrm{O}\right) ; \delta 4.96\left(\mathrm{Z}-\mathrm{CH}_{2}\right) ; \delta$ 7.05-7.40 $\left(\mathrm{C}_{6} \mathrm{H}_{5}+\alpha \mathrm{CH}-\left(\mathrm{CH}_{2}\right)_{4}-\mathrm{NH}+\mathrm{S}-\left(\mathrm{CH}_{2}-\mathrm{CH}-\mathrm{N} H\right) ; \delta\right.$ 7.67-8.34 $\left(\alpha \mathrm{CH}-\mathrm{NH}+\mathrm{CH}_{2}-\mathrm{NH}-(\mathrm{C}=\mathrm{O})-\mathrm{CH}-\right.$ S).

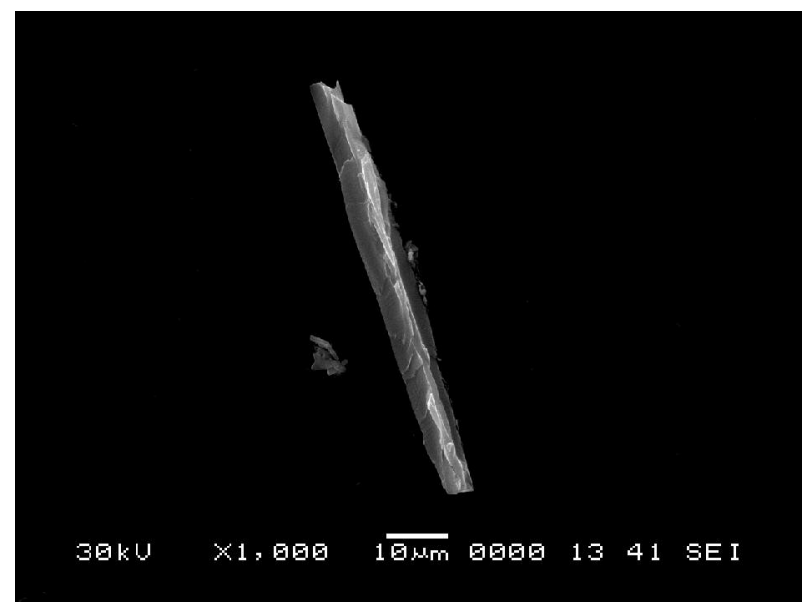

Figure S1. Scanning electron micrograph of deprotected lipopolypeptide IV-2. 